\title{
A Method of Go Recognition Based on Deep Learning
}

\author{
Heng Ran ${ }^{a}$, Pengyun Song ${ }^{b,}{ }^{*}$, Yanghui Liu ${ }^{c}$, Lei Yu ${ }^{d}$, Hang Zhou ${ }^{e}$ and \\ Yinrui Zhang ${ }^{f}$
}

Southwest Minzu University, Chengdu 610000, China.

a510589395@qq.com, bsongpy23@foxmail.com, c1479814320@qq.com,

d13773006730@163.com, e540308889@qq.com, f1162781443@qq.com

Keywords: Deep learning, go, image processing.

\begin{abstract}
In order to obtain the image information of go, we use the traditional method to process the image, but the traditional method has great limitation. The accuracy is low, and the external factors can easily affect the processing result. In order to solve the limitation of traditional image processing method, we will introduce and study a new image processing method-depth learning. First, we prepare a picture sample of go, and then we use two methods to identify the image of go. Finally, the recognition results of the traditional method and the recognition results of the depth learning are compared. The results show that the accuracy of the depth learning method is improved by $17.1 \%$ compared with the traditional method.
\end{abstract}

\section{Introduction}

As early as the last century, the theory of deep learning has been put forward. In recent years, artificial intelligence has become a subject area with many practical applications and active research, and it is booming. And deep learning is one of the ways to artificial intelligence. It is a kind of machine learning, a technology that enables computer systems to be improved from experience and data, with powerful capabilities and flexibility, and defines complex concepts by the connections between simple concepts. From general abstraction to high-level abstract representation.

With the continuous progress and development of computer industry, the theory of deep learning has been applied in many aspects, and gradually formed, which makes it more and more popular. In the robotics industry to improve the system's self-learning efficiency and adaptability; also promoted the rapid development of artificial intelligence; from Google Cat to Baidu brain; Speech recognition, big data and so on. Image processing is a very important function of deep learning. This paper will introduce the differences between traditional methods and deep learning, as well as the advantages of deep learning in the recognition of go black and white and chessboard. First, the traditional method of identifying images is introduced:

The traditional method of detecting chessboard and black and white chess (Fig. 1: traditional method flowchart):

The first step is to collect images: take pictures and collect pictures on the chessboard;

The second step, image grayscale processing: because the image processing is easy to process the grayscale image, carries on the grayscale processing to the picture, transforms the color image which the mobile phone uploads into the gray image;

The third step, edge detection: the gray image edge detection, gray image edge contour detection, and the binary image display;

The fourth step, high pass filter: the binary image is filtered, and the noise in the image is filtered by high pass filter to get a better binary image.

The fifth step, Hough transform: the slope and intercept of the row and column in the image are detected by Hough straight line change;

The sixth step, Line fitting: straight line fitting is the approximation of scattered points in an image to fit a straight line. RANSAC algorithm can approximate the true value to filter out the influence of 
abnormal points. The RANSAC algorithm is used to fit the row and column straight line of the board to get the complete board.

The seventh steps, chess color recognition: the k-means clustering algorithm is used to recognize the chess color. The clustering algorithm is actually the collection of similar elements. There are also classification problems in image processing. Cluster analysis is also called cluster analysis. It is a statistical analysis method to study the classification problem. It is also an important algorithm of data mining. The $\mathrm{k}$-means algorithm accepts the input amount as $\mathrm{k}$, and then divides $\mathrm{n}$ data into $\mathrm{k}$ classes. In order to meet the high similarity in the same class, but not in the same class, the similarity is small. Similarity is calculated by the central object obtained from the average value of the objects in each class.

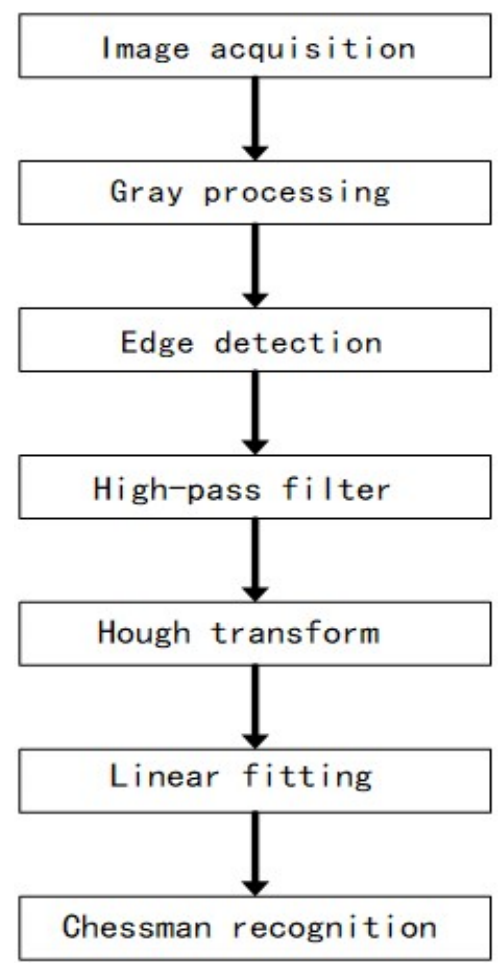

Figure 1: Traditional Method Flowchart

The method to detect the checkerboard and the color of pieces by deep learning method is as follows:

The first step is to make and download training data sets; The training of the neural network is based on a large number of pictures of go. Due to the small number of online go pictures, it is necessary to shoot or find a part of it and download some of it online.

The second step is to preprocess the images in the training data set. Neural network training needs specific specifications of the picture, every picture should be standard size, and scaling to $224 \times 224$ images, and the standard size image and its corresponding category save in pairs, a class of "standard size image " as a data.

The third step is to construct and train the convolution neural network model. Create multiple hidden layers for training, similar to neurons processing information. Successive computation layer alternate between convolution and sampling, we get the effect of a "double spire", is in each convolution or sampling layer, reduced with the spatial resolution, and the corresponding increase in the number compared to the previous layer feature mapping. The idea of subsampling after convolution is inspired by the idea of "simple" cells in the animal vision system followed by "complex" cells.

The fourth step is to select the test photo set, carry out the test, and test the effect.

Compare the results of the two methods, as shown in figure 2 and figure 3:(Figure 2 is a traditional method rendering. Figure 3 shows the effect diagram of deep learning;)

As can be seen from the traditional method in figure 2, there are also some black and white pieces that are not identified in the figure, which is the chess pieces without the box in the picture. From the 
depth study of figure 3, it can be found that the pieces in the picture have been accurately identified. Such results are also obtained through a large number of test data, and figure 2 and figure 3 are the most common. Therefore, we find that the method of deep learning can identify the information in the picture more steadily and accurately, and feedback the detected data accurately. Through a large number of data training neural network, the detection of deep learning method can not be affected by many external factors, such as uneven illumination, strong illumination, bad shooting Angle and so on. Deep learning has many advantages, such as DL training process integrating feature extraction; Collection of large data sets; Technological progress and innovation and so on. Thus, deep learning to identify pieces is a more accurate, more efficient and up-to-date method.

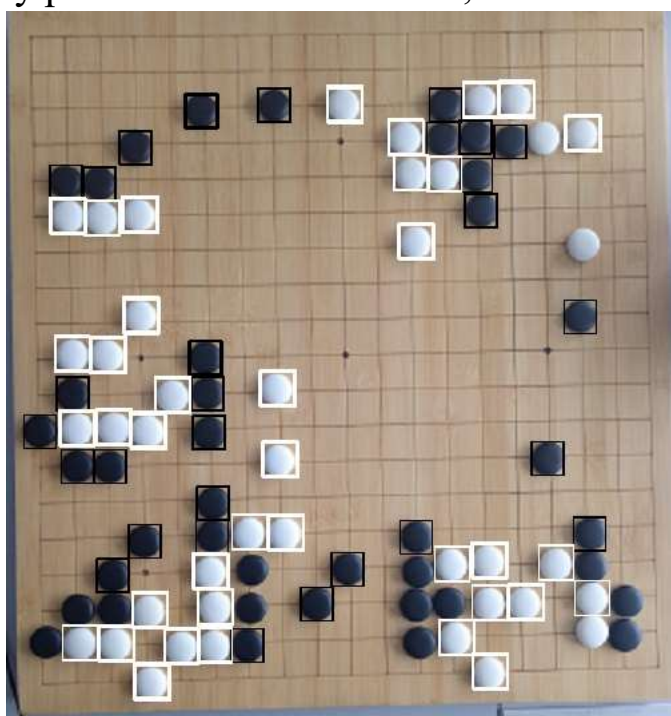

Figure 2 Traditional methods

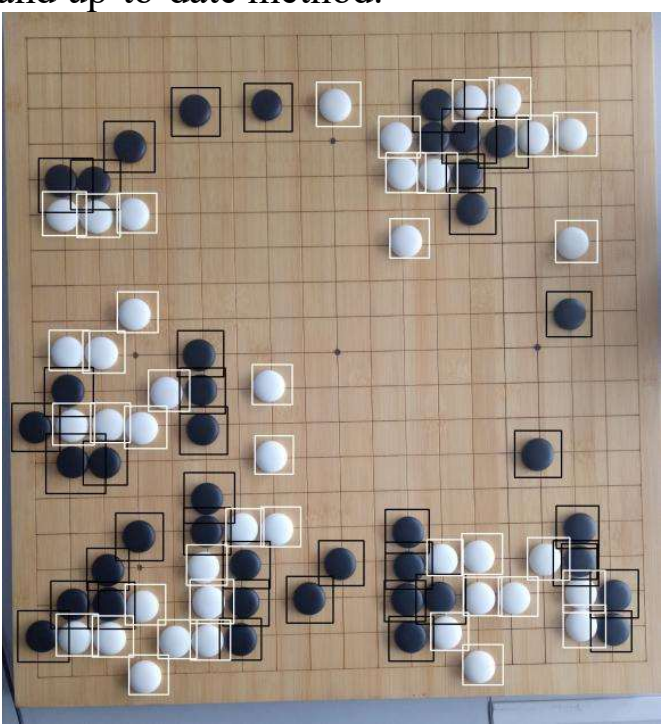

Figure 3 Deep learning method

\section{Conclusion}

The in-depth learning approach solves many of the problems of traditional methods:

(1) The results are not affected by many external factors, such as uneven illumination, strong illumination, poor shooting angle and so on.

(2) More stable, high-precision recognition of the information in the picture.

(3) Fewer peripherals are needed.

(4) The recognition accuracy increased by $17.1 \%$.

\section{Acknowledgements}

The First Author: Heng Ran. The research direction is digital image processing.

The Corresponding Author: Pengyun Song. The research direction is digital image processing.

Undergraduate Entrepreneurship and Innovation Project(201710656002)

\section{References}

[1]. Li Guo, Zeilin Gao, Yu Liao. An improved RANSAC image matching algorithm. Communication and information technology phase 3, 2014.

[2]. Shengyong Chen, Sheng Liu. Computer vision technology based on OpenCV. Science press,2008(18), p.141-142.

[3]. Huan Zhang: algorithm research on the color of grayscale images (master, xi 'an university of electronic science and technology, China, 2015).

[4]. Shuai Tong. Image edge detection algorithm research. Computer optical disk software and application, 2013. 
[5]. Wentai Qu. Elliptical detection method based on Hough transform in the midpoint of the string. Journal of zhejiang university (engineering edition), 2005.

[6]. Jigui Sun, Jie Liu, Lianyu Zhao. Clustering algorithm research. Software journal, 2008, p.48-61.

[7]. Han Liu, Lin He, Jun Li. Deep learning progress and its application in image processing. Zte technology, 2017, p.36-40.

[8]. Jie Lin, Ruyi Li. Image recognition processing based on deep learning. Network security technology and application, 2017, p.65-67.

[9]. Yuyan Wang. Application and development of deep learning in image recognition. China's strategic emerging industries, 2017.

[10]. Zhengping Gu, Min Zhu. Research on fish classification algorithm based on deep learning. Computer application and software, 2018, p.200-205.

[11]. Alex Krizhevsky,Alex Krizhevsky,Geoffrey E.Hinton.ImageNet Classification with Deep Convolutional Neural Networks.International Conference on Neural Information Processing Systems,2012,p.1097-1105.

[12]. Bingyuan Liu. Study on feature representation and image classification method based on deep learning.2015. 